\title{
Factors influencing breast elasticity measured by the ultrasound Shear Wave elastography - preliminary results
}

Paweł Rzymski1 ${ }^{1}$ Agnieszka Skórzewska², Myriam Skibińska-Zielińska², Tomasz Opala ${ }^{1}$

${ }^{1}$ Department of Mother's and Child's Health, University of Medical Sciences, Gynecologic and Obstetrical University Hospital, Poznan, Poland

2Department of Radiology, University of Medical Sciences, Gynecologic and Obstetrical University Hospital, Poznan, Poland

Submitted: 11 June 2010

Accepted: 31 July 2010

Arch Med Sci 2011; 7, 1: 127-133

DOI: 10.5114 /aoms.2011.20617

Copyright () 2011 Termedia \& Banach

\begin{abstract}
Introduction: Many physiological changes of breast elasticity depend on the age, hormonal status, menstrual cycle and many others. The aim of this study was to evaluate viscoelastic properties of normal breast tissues in a large group of women and to search for factors which play a role in its mechanical properties. Material and methods: 101 women aged 18-74 years who underwent B-mode sonography and additionally sonoelastography. We measured viscoelasticity in 8 quadrants by a share wave ultrasonic device estimating Young modules in regions of interest.

Results: Mean elasticity measured in all 8 scans in glandular and fatty tissue were $11.28 \pm 5.79 \mathrm{kPa}(0.1-46.26 \mathrm{kPa})$ and $9.24 \pm 4.48 \mathrm{kPa}(0.1-29.78 \mathrm{kPa})$, respectively. The correlation between age and mean elasticity of glandular tissue was $R s=0.27(p=0.007)$. The correlation between glandular tissue elasticity heterogeneity and breast mastalgia measured by VAS was $R s=-0.23(p=0.241)$. Fat tissue elasticity correlated with duration of lactation was $R s=0.21(p=0.01)$. Conclusions: There are several parameters influencing breast viscoelasticity measured by share wave sonoelastography. Glandular tissue elasticity correlates positively with age, fatty tissue elasticity correlates positively with duration of lactation and heterogeneity of the elasticity map of glandular tissue correlates with breast pain and fat tissue with BMI.
\end{abstract}

Key words: elastography, breast elasticity, breast ultrasound, viscoelasticity, share wave elastography.

\section{Introduction}

The mechanics of normal breast in women is complicated and there is no literature in this field. The knowledge of breast forces, vectors and tissue mechanics is very important for clinical applications like planning in plastic surgery, breast cancer risk identification and prediction and others [1].

Breast tissue composition is a very important risk factor for breast cancer. Techniques evaluating the breast as a mixture of tissues like: mammography, ultrasonography, magnetic resonance imaging (MRI) or elastography are methods of evaluating a histology-like map of tissues [2]. The two major tissues building the breast are glandular and fatty tissue. Their proportions, connective tissue content, size, shape also vary over the time in the same woman (e.g. during the menstrual cycle) and between women $[1,3,4]$. There are many studies indicating that the increase in

\author{
Corresponding author: \\ Paweł Rzymski MD, PhD \\ Department of Mother's \\ and Child's Health \\ University of Medical Sciences \\ Gynecologic and Obstetrical \\ University Hospital \\ Polna St 33, Poznań, Poland \\ Phone: +48 605393096 \\ Fax: +48 618419618 \\ E-mail: \\ parzymsk@gpsk.am.poznan.pl
}


breast density as measured e.g. in mammography, is a strong risk factor for breast cancer. Efforts are also made to quantify the breast density $[5,6]$. Since Wolfe divided the breast density according to the cancer risk, a variety of approaches for measuring breast density was developed. Most of these quantitative measures ignore the physical 3D features of the breast. Breasts have different thickness and potentially could yield the same mammography density corresponding to widely varying volumetric breast density values [6].

Such limitation does not appear when measuring breast elasticity by ultrasound elastography. Breast viscoelasticity measurement, especially by newly developed sonoelastography techniques, is suggested to be important for breast tumour diagnosis. Elasticity could be also a potential risk factor for breast cancer similar to breast density measured by other imaging techniques. It remains an open question how elasticity corresponds to breast fibroglandular density. The aim of this study was to evaluate viscoelastic properties of normal breast tissues in a larger group of women and to search for anamnestic factors which play a role in its mechanical properties.

\section{Material and methods}

We examined 101 women aged 18-74 years who underwent B-mode sonography in the Gynaecologic and Obstetrical University Hospital in Poznań, Poland. The study was performed between November 2009 and April 2010. All subjects filled in the questionnaire concerning a general medical, obstetrical and breast history as well as Visual Analogue Scale (VAS) of breast cyclic or non-cyclic mastalgia. Among subjects, 47 women underwent screening mammography according to national guidelines and additionally underwent sonoelastography. Their examinations yielded no abnormalities (BIRADS 1, Breast Imaging Reporting and Data System 1). Other 54 patients were diagnosed in our departments because of breast disorders including: breast pain, breast palpable mass or were healthy volunteers. All 101 patients underwent classical B-mode breast sonography (Aixplorer Ultrasound System, SuperSonic Imagine SA, France), which yielded no abnormalities

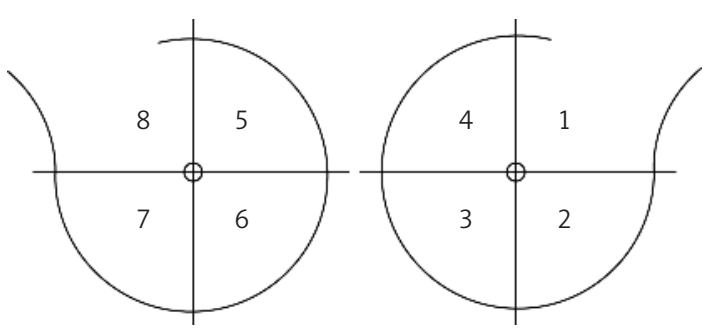

Figure 1. Division of eight scans taken during the breast elasticity ultrasonic examination
(BIRADS $1, n=56$ ) or benign cysts, tumours and other benign disorders, classified as BIRADS 2 $(n=42)$ and BIRADS $3(n=3)$. The ultrasound examination as well as sonoelastography were performed by a gynaecologist with 7 years' experience in breast ultrasound (P.R.) and radiologists (A.S. and M.S-Z) with 16 and 14 years' experience in breast ultrasound, respectively. The equipment enables performance of normal ultrasound with linear probe $(15 \mathrm{MHz})$ and additionally real-time sonoelastography. The methodology consists of the generation of a remote radiation force by focused ultrasonic beams. Each pushing beam generates remote vibration that results in the propagation of transient shear wave. Several pushing beams transmitted at different depths result in quasi-plane shear wave front which propagates through the whole imaging region of interest (ROI) [7, 8]. After the B-mode ultrasound, a second part of examination was performed, which was the elastography research protocol. We divided the left and right breast into 8 quadrants and obtained, using the elastography mode, 8 scans of normal breast tissue from both breasts in the order illustrated in Figure 1. In a group of patients classified as BIRADS 2 or 3 we omitted the pathologic region which was previously diagnosed (e.g. cysts) with a distance of minimum $1.5 \mathrm{~cm}$.

When obtaining a single scan the region of interest (ROI) was set to include subcutaneous fat at the top and the margin of pectoral muscle at the bottom. The best grayscale scan was used to identify glandular and fat tissue. All elastography scans included measurements with 2 to $3 \mathrm{~mm}$ diameter Q-box (area of elasticity measurement in $\mathrm{kPa}$ ) in glandular and fat tissue. The chosen area was typical and representative for glandular and fatty tissue in grayscale ultrasound and Q-box diameter depended on the tissue diameters. Parameters included mean elasticity in Q-box, minimal and maximal elasticity in Q-box, standard deviation (SD). Additionally, we calculated the heterogeneity of the sonoelasticity map in ROI by estimating a minimum to maximum range and dividing it by the minimal elasticity value and mean value. From all 101 patients, 808 elastography scans were obtained from 8 quadrants. For analysis, we calculated a glandular to fatty tissue ratio and we also grouped breast quadrants into inner (scans 3 , 4, 5 and 6) and outer ones (scans 1, 2, 7 and 8).

All data were stored in Ms Excel calculation sheet (Microsoft Corp, USA) and analyzed with SigmaStat 3.1 (Jandel Corp, USA). The normal distribution was confirmed by Kolgomorov-Smirnoff test. Differences between parameters were analyzed with MannWhitney test. The correlation between parameters was checked by Spearman's test. The factor influencing elasticity (dependent value) was 
checked by the multiple linear regression model. $P$ values less than 0.05 were considered significant. The study was approved by a local bioethics committee at the Poznań University of Medical Sciences.

\section{Results}

Mean age of the examined group was $43.1 \pm 11.6$ years. Mean elasticity measured in all 8 scans in glandular and fatty tissue was $11.28 \pm 5.79 \mathrm{kPa}$ (0.1-46.26 kPa) and 9.24 $\pm 4.48 \mathrm{kPa}(0.1-29.78 \mathrm{kPa})$, respectively. The correlation between glandular and fat tissue and age, pregnancies, lactation, BMI (Body Mass Index) and mastalgia is presented in Table I. When dividing breast quadrants into inner and outer quadrants, some differences in elasticity were noticed. Mean elasticity values in glandular tissue in inner quadrants vs. outer quadrants were 12.4 \pm 6.4 and $10.9 \pm 8.3$, respectively $(p<0.001)$. Mean elasticity values in fat tissue in inner quadrants vs. outer quadrants were $10.6 \pm 5.3$ and $8.1 \pm 4.7$, respectively $(p<0.001)$. The correlation between glandular and fat tissue according to inner and outer quadrants and age, pregnancies, lactation, $\mathrm{BMI}$ and mastalgia is presented in Table II.

The mean elasticity heterogeneity of glandular tissue was $6.22 \pm 3.48 \mathrm{kPa}(0-21.2 \mathrm{kPa})$. Mean elasticity heterogeneity of glandular tissue in inner and outer quadrants was $7.09 \pm 4.35 \mathrm{kPa}(0-29.5 \mathrm{kPa})$ and $5.35 \pm 3.79 \mathrm{kPa}(0-21.2 \mathrm{kPa})$, respectively.

Table I. Correlations between glandular and fat tissue elasticity and selected parameters

\begin{tabular}{|c|c|c|c|c|c|c|}
\hline & $\begin{array}{c}\text { Mean } \\
\text { glandular } \\
\text { elasticity } \\
\text { of both } \\
\text { breasts }[\mathrm{kPa}]\end{array}$ & $\begin{array}{c}\text { Minimum } \\
\text { glandular } \\
\text { elasticity } \\
\text { of both } \\
\text { breasts [kPa] }\end{array}$ & $\begin{array}{c}\text { Maximum } \\
\text { glandular } \\
\text { elasticity } \\
\text { of both } \\
\text { breasts [kPa] }\end{array}$ & $\begin{array}{c}\text { Mean fatty } \\
\text { tissue } \\
\text { elasticity } \\
\text { of both } \\
\text { breasts [kPa] }\end{array}$ & $\begin{array}{c}\text { Minimum } \\
\text { fatty tissue } \\
\text { elasticity } \\
\text { of both } \\
\text { breasts [kPa] }\end{array}$ & $\begin{array}{c}\text { Maximum } \\
\text { fatty tissue } \\
\text { elasticity } \\
\text { of both } \\
\text { breasts [kPa] }\end{array}$ \\
\hline Age [years] & $\begin{array}{l}R s=0.27 \\
P=0.007\end{array}$ & $\begin{array}{l}R s=0.24 \\
P=0.0172\end{array}$ & $\begin{array}{l}R s=0.22 \\
p=0.0321\end{array}$ & $\begin{array}{l}R s=0.10 \\
P=0.30\end{array}$ & $\begin{array}{c}R s=0.13 \\
p=0.18\end{array}$ & $\begin{array}{c}R s=0.01 \\
p=0.91\end{array}$ \\
\hline Pregnancies [No] & $\begin{array}{l}R s=0.01 \\
P=0.84\end{array}$ & $\begin{array}{c}R s=0.04 \\
p=0.67\end{array}$ & $\begin{array}{l}R s=0.01 \\
p=0.88\end{array}$ & $\begin{array}{l}R s=0.05 \\
P=0.62\end{array}$ & $\begin{array}{l}R s=0.06 \\
p=0.50\end{array}$ & $\begin{array}{l}R s=0.03 \\
p=0.76\end{array}$ \\
\hline $\begin{array}{l}\text { Age of the } 1^{\text {st }} \\
\text { delivery [years] }\end{array}$ & $\begin{aligned} R s & =0.06 \\
P & =0.53\end{aligned}$ & $\begin{array}{l}R s=0.10 \\
p=0.32\end{array}$ & $\begin{array}{c}R s=0.09 \\
p=0.37\end{array}$ & $\begin{array}{l}R s=0.11 \\
P=0.27\end{array}$ & $\begin{array}{c}R s=0.14 \\
p=0.17\end{array}$ & $\begin{array}{c}R s=0.15 \\
p=0.14\end{array}$ \\
\hline Lactation [years] & $\begin{array}{l}R s=0.01 \\
P=0.86\end{array}$ & $\begin{array}{l}R s=0.11 \\
p=0.27\end{array}$ & $\begin{array}{c}R s=0.03 \\
p=0.76\end{array}$ & $\begin{array}{l}R s=0.21 \\
P=0.03\end{array}$ & $\begin{array}{l}R s=0.25 \\
p=0.01\end{array}$ & $\begin{array}{c}R s=0.25 \\
p=0.01\end{array}$ \\
\hline $\mathrm{BMI}\left[\mathrm{kg} / \mathrm{m}^{2}\right]$ & $\begin{array}{c}R s=0.19 \\
P=0.55\end{array}$ & $\begin{array}{l}R s=0.09 \\
p=0.32\end{array}$ & $\begin{array}{l}R s=0.10 \\
p=0.29\end{array}$ & $\begin{array}{c}R s=0.09 \\
P=0.33\end{array}$ & $\begin{array}{l}R s=0.10 \\
p=0.30\end{array}$ & $\begin{aligned} R s & =-0.02 \\
p & =0.79\end{aligned}$ \\
\hline $\begin{array}{l}\text { VAS breast pain } \\
{[\mathrm{mm}]}\end{array}$ & $\begin{aligned} R s & =-0.13 \\
P & =0.19\end{aligned}$ & $\begin{array}{c}R s=0.04 \\
p=0.70\end{array}$ & $\begin{aligned} R s & =-0.05 \\
p & =0.60\end{aligned}$ & $\begin{aligned} R s & =-0.12 \\
P & =0.21\end{aligned}$ & $\begin{aligned} R s & =-0.01 \\
p & =0.87\end{aligned}$ & $\begin{aligned} R s & =-0.11 \\
p & =0.31\end{aligned}$ \\
\hline
\end{tabular}

Table II. Correlations between elasticity divided into inner and outer quadrants and the glandular to fat elasticity ratio and selected parameters

\begin{tabular}{|c|c|c|c|c|c|c|}
\hline & $\begin{array}{c}\text { Mean } \\
\text { glandular } \\
\text { elasticity } \\
\text { of inner } \\
\text { quadrants }[\mathrm{kPa}]\end{array}$ & $\begin{array}{c}\text { Mean } \\
\text { glandular } \\
\text { elasticity } \\
\text { of outer } \\
\text { quadrants }[\mathrm{kPa}]\end{array}$ & $\begin{array}{c}\text { Mean fatty } \\
\text { tissue } \\
\text { elasticity } \\
\text { of inner } \\
\text { quadrants [kPa] }\end{array}$ & $\begin{array}{l}\text { Mean fatty } \\
\text { tissue } \\
\text { elasticity } \\
\text { of outer } \\
\text { quadrants [kPa] }\end{array}$ & $\begin{array}{c}\text { Glandular to } \\
\text { fat ratio (G/L) } \\
\text { elasticity } \\
\text { of inner } \\
\text { quadrants [1/1] }\end{array}$ & $\begin{array}{c}\text { Glandular to } \\
\text { fat ratio (G/L) } \\
\text { elasticity } \\
\text { of outer } \\
\text { quadrants [1/1] }\end{array}$ \\
\hline Age [years] & $\begin{array}{l}R s=0.32 \\
P=0.001\end{array}$ & $\begin{array}{c}R S=0.14 \\
P=0.16\end{array}$ & $\begin{array}{c}R s=0.12 \\
p=0.34\end{array}$ & $\begin{array}{c}R s=0.04 \\
p=0.64\end{array}$ & $\begin{aligned} R s & =0.16 \\
p & =0.11\end{aligned}$ & $\begin{array}{l}R s=0.24 \\
p=0.0155\end{array}$ \\
\hline Pregnancies [No] & $\begin{array}{c}R s=0.09 \\
p=0.38\end{array}$ & $\begin{aligned} R s & =-0.04 \\
p & =0.68\end{aligned}$ & $\begin{aligned} R s & =0.08 \\
p & =0.43\end{aligned}$ & $\begin{aligned} R s & =-0.01 \\
p & =0.89\end{aligned}$ & $\begin{aligned} R s & =0.003 \\
p & =0.97\end{aligned}$ & $\begin{array}{c}R s=0.58 \\
p=0.57\end{array}$ \\
\hline $\begin{array}{l}\text { Age of the } 1^{\text {st }} \\
\text { delivery [years] }\end{array}$ & $\begin{aligned} R s & =0.09 \\
p & =0.38\end{aligned}$ & $\begin{aligned} R s & =0.05 \\
p & =0.63\end{aligned}$ & $\begin{array}{l}R s=0.18 \\
p=0.07\end{array}$ & $\begin{array}{c}R s=0.02 \\
p=0.79\end{array}$ & $\begin{aligned} R s & =-0.19 \\
p & =0.05\end{aligned}$ & $\begin{array}{c}R s=0.05 \\
p=0.64\end{array}$ \\
\hline Lactation [years] & $\begin{array}{l}R s=0.05 \\
p=0.65\end{array}$ & $\begin{aligned} R s & =0.00 \\
p & =0.99\end{aligned}$ & $\begin{array}{l}R s=0.28 \\
p=0.005\end{array}$ & $\begin{aligned} R s & =0.07 \\
p & =0.51\end{aligned}$ & $\begin{array}{l}R s=-0.26 \\
p=0.0121\end{array}$ & $\begin{aligned} R s & =-0.15 \\
p & =0.15\end{aligned}$ \\
\hline BMI $\left[\mathrm{kg} / \mathrm{m}^{2}\right]$ & $\begin{array}{c}R s=0.15 \\
p=0.14\end{array}$ & $\begin{array}{l}R s=0.66 \\
p=0.50\end{array}$ & $\begin{aligned} R s & =0.06 \\
p & =0.56\end{aligned}$ & $\begin{aligned} R s & =-0.01 \\
p & =0.90\end{aligned}$ & $\begin{array}{c}R s=0.08 \\
p=0.42\end{array}$ & $\begin{array}{c}R s=0.22 \\
p=0.0274\end{array}$ \\
\hline $\begin{array}{l}\text { VAS breast pain } \\
{[\mathrm{mm}]}\end{array}$ & $\begin{aligned} R s & =-0.07 \\
p & =0.53\end{aligned}$ & $\begin{array}{c}R s=0.15 \\
p=0.16\end{array}$ & $\begin{aligned} R s & =-0.07 \\
p & =0.50\end{aligned}$ & $\begin{array}{c}R s=0.05 \\
p=0.62\end{array}$ & $\begin{aligned} R s & =-0.03 \\
p & =0.77\end{aligned}$ & $\begin{aligned} R s & =0.08 \\
p & =0.42\end{aligned}$ \\
\hline
\end{tabular}


Table III. Analysis of elasticity heterogeneity in ROI with selected parameters

\begin{tabular}{|c|c|c|c|c|c|c|}
\hline & $\begin{array}{c}\text { Age } \\
\text { [years] }\end{array}$ & $\begin{array}{l}\text { Pregnancies } \\
{[\mathrm{No}]}\end{array}$ & $\begin{array}{c}\text { Age of the } \\
1^{\text {st }} \text { delivery [years] }\end{array}$ & $\begin{array}{l}\text { Lactation } \\
\text { [months] }\end{array}$ & $\begin{array}{c}\mathrm{BMI} \\
{\left[\mathrm{kg} / \mathrm{m}^{2}\right]}\end{array}$ & $\begin{array}{l}\text { VAS breast } \\
\text { pain }[\mathrm{mm}]\end{array}$ \\
\hline $\begin{array}{l}\text { Glandular tissue } \\
\text { heterogeneity } \\
\text { (max-min, } \mathrm{kPa})\end{array}$ & $\begin{array}{c}R s=0.13 \\
p=0.19\end{array}$ & $\begin{aligned} R s & =0.00 \\
p & =0.93\end{aligned}$ & $\begin{aligned} R s & =0.08 \\
p & =0.41\end{aligned}$ & $\begin{aligned} R s & =-0.03 \\
p & =0.70\end{aligned}$ & $\begin{aligned} R s & =0.09 \\
p & =0.36\end{aligned}$ & $\begin{aligned} R s & =-0.15 \\
p & =0.14\end{aligned}$ \\
\hline $\begin{array}{l}\text { Glandular tissue } \\
\text { heterogeneity } \\
\text { (max-min, kPa), } \\
\text { inner quadrants }\end{array}$ & $\begin{array}{l}R s=0.09 \\
p=0.32\end{array}$ & $\begin{array}{l}R s=0.04 \\
p=0.65\end{array}$ & $\begin{array}{l}R s=0.08 \\
p=0.40\end{array}$ & $\begin{aligned} R s & =-0.05 \\
p & =0.58\end{aligned}$ & $\begin{aligned} R s & =-0.01 \\
p & =0.93\end{aligned}$ & $\begin{aligned} R s & =-0.16 \\
p & =0.15\end{aligned}$ \\
\hline $\begin{array}{l}\text { Glandular tissue } \\
\text { heterogeneity } \\
\text { (max-min, } \mathrm{kPa} \text { ), } \\
\text { outer quadrants }\end{array}$ & $\begin{array}{l}R s=0.14 \\
p=0.14\end{array}$ & $\begin{aligned} R s & =-0.02 \\
p & =0.83\end{aligned}$ & $\begin{aligned} R s & =0.09 \\
p & =0.35\end{aligned}$ & $\begin{aligned} R s & =0.02 \\
p & =0.81\end{aligned}$ & $\begin{array}{l}R s=0.17 \\
p=0.07\end{array}$ & $\begin{aligned} R s & =-0.11 \\
p & =0.33\end{aligned}$ \\
\hline $\begin{array}{l}\text { Glandular tissue } \\
\text { heterogeneity } \\
\text { (\% of mean elasticity) }\end{array}$ & $\begin{aligned} R s & =-0.13 \\
p & =0.18\end{aligned}$ & $\begin{aligned} R s & =-0.04 \\
p & =0.65\end{aligned}$ & $\begin{aligned} R s & =-0.01 \\
p & =0.94\end{aligned}$ & $\begin{aligned} R s & =-0.15 \\
p & =0.13\end{aligned}$ & $\begin{aligned} R s & =-0.01 \\
p & =0.89\end{aligned}$ & $\begin{array}{l}R s=-0.23 \\
p=0.0241\end{array}$ \\
\hline $\begin{array}{l}\text { Glandular tissue } \\
\text { heterogeneity } \\
\text { (\% of minimal elasticity) }\end{array}$ & $\begin{aligned} R s & =-0.12 \\
p & =0.21\end{aligned}$ & $\begin{aligned} R s & =-0.03 \\
p & =0.76\end{aligned}$ & $\begin{array}{l}R s=0.01 \\
p=0.96\end{array}$ & $\begin{aligned} R s & =-0.13 \\
p & =0.17\end{aligned}$ & $\begin{aligned} R s & =-0.01 \\
p & =0.91\end{aligned}$ & $\begin{array}{l}R s=-0.25 \\
p=0.0226\end{array}$ \\
\hline $\begin{array}{l}\text { Fat tissue heterogeneity } \\
\text { (max-min, } \mathrm{kPa} \text { ) }\end{array}$ & $\begin{aligned} R s & =-0.03 \\
p & =0.78\end{aligned}$ & $\begin{array}{l}R s=0.01 \\
p=0.92\end{array}$ & $\begin{array}{l}R s=0.12 \\
p=0.22\end{array}$ & $\begin{array}{l}R s=0.17 \\
p=0.08\end{array}$ & $\begin{aligned} R s & =-0.09 \\
p & =0.37\end{aligned}$ & $\begin{array}{l}R s=-0.21 \\
p=0.055\end{array}$ \\
\hline $\begin{array}{l}\text { Fat tissue heterogeneity } \\
\text { (max-min, } \mathrm{kPa} \text { ), } \\
\text { inner quadrants }\end{array}$ & $\begin{aligned} R s & =-0.01 \\
p & =0.96\end{aligned}$ & $\begin{aligned} R s & =0.03 \\
p & =0.71\end{aligned}$ & $\begin{array}{l}R s=0.18 \\
p=0.06\end{array}$ & $\begin{array}{c}R s=0.22 \\
p=0.0318\end{array}$ & $\begin{aligned} R s & =-0.10 \\
p & =0.32\end{aligned}$ & $\begin{array}{l}R s=-0.29 \\
p=0.007\end{array}$ \\
\hline $\begin{array}{l}\text { Fat tissue heterogeneity } \\
\text { (max-min, } \mathrm{kPa}) \\
\text { outer quadrants }\end{array}$ & $\begin{aligned} R s & =-0.01 \\
p & =0.92\end{aligned}$ & $\begin{array}{l}R s=0.01 \\
p=0.90\end{array}$ & $\begin{array}{c}R s=0.08 \\
p=0.44\end{array}$ & $\begin{aligned} R s & =0.09 \\
p & =0.34\end{aligned}$ & $\begin{aligned} R s & =-0.08 \\
p & =0.40\end{aligned}$ & $\begin{aligned} R s & =0.03 \\
p & =0.77\end{aligned}$ \\
\hline $\begin{array}{l}\text { Fat tissue heterogeneity } \\
\text { (\% of mean elasticity) }\end{array}$ & $\begin{aligned} R s & =-0.17 \\
p & =0.08\end{aligned}$ & $\begin{aligned} R s & =-0.01 \\
p & =0.93\end{aligned}$ & $\begin{aligned} R s & =0.05 \\
p & =0.64\end{aligned}$ & $\begin{aligned} R s & =-0.05 \\
p & =0.62\end{aligned}$ & $\begin{aligned} R s & =-0.24 \\
p & =0.016\end{aligned}$ & $\begin{aligned} R s & =-0.16 \\
p & =0.14\end{aligned}$ \\
\hline $\begin{array}{l}\text { Fat tissue heterogeneity } \\
\text { (\% of minimal elasticity) }\end{array}$ & $\begin{aligned} R s & =-0.18 \\
p & =0.08\end{aligned}$ & $\begin{array}{l}R s=0.01 \\
p=0.94\end{array}$ & $\begin{aligned} R s & =0.04 \\
p & =0.70\end{aligned}$ & $\begin{aligned} R s & =-0.05 \\
p & =0.59\end{aligned}$ & $\begin{array}{l}R s=-0.22 \\
p=0.0256\end{array}$ & $\begin{aligned} R s & =-0.15 \\
p & =0.16\end{aligned}$ \\
\hline
\end{tabular}

Heterogeneity of glandular tissue expressed as $\%$ of mean elasticity was $55.1 \pm 18.0 \%$ (0-103.8\%). The mean elasticity heterogeneity of fat tissue was $5.52 \pm 3.04 \mathrm{kPa}(0-17.2 \mathrm{kPa})$. Mean elasticity

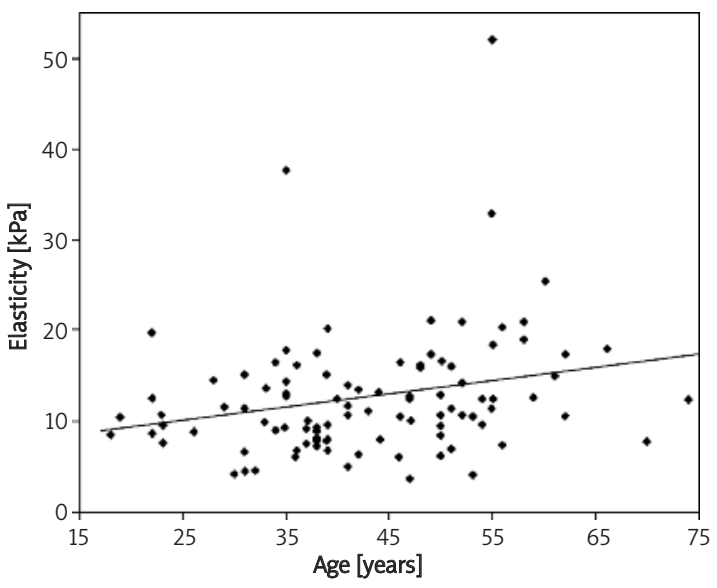

Figure 2. Correlation between age and mean elasticity of glandular tissue measured in quadrants 1-8 (both breasts) heterogeneity of fat tissue in inner and outer quadrants was $6.21 \pm 3.86 \mathrm{kPa}(0-19.8 \mathrm{kPa})$ and 4.82 $\pm 3.20 \mathrm{kPa}(0-17.0 \mathrm{kPa})$, respectively. Heterogeneity of fat tissue expressed as \% of mean elasticity was $61.0 \% \pm 24.4$ (0-144.9\%). The difference in heterogeneity between glandular and fat tissue in the whole breast, inner quadrants, outer quadrants was statistically insignificant $(p=0.079, p=0.084$, $p=0.026$, respectively). Correlations between breast elasticity heterogeneity and selected parameters are presented in Table III and Figuers 2-4.

We also performed the regression analysis. The best fit model involving two parameters (age and duration of lactation) explaining $15 \%$ of elasticity results in our study (adjusted $R^{2}=0.146$ ) was: glandular tissue elasticity $(\mathrm{kPa})=1.011+(0.0132$ $\times$ age $)-(0.0143 \times$ months of lactation $) \pm 0.451$.

\section{Discussion}

The main purpose of this study was to evaluate elasticity of normal breast tissues in vivo using 
supersonic share imaging. Morphologic scoring of breast elasticity maps parallel to BI-RADS classification was suggested by some authors. Many papers analyzed the possible use of Matsumura elasticity scoring system in differential diagnosis of breast tumours, which had higher sensitivity and specificity than B-mode ultrasound $[9,10]$. In the major part of elastography studies the precise quantification of elasticity was not possible because the elasticity map represented relative values during freehand breast compression $[9,11]$. Using the new shear wave technology, the exact quantification of elasticity (or precisely to say Young modules) is possible as well as its colourcoded map [8]. Normal breast elasticity evaluation is still missing in the literature $[9,11]$. We and other authors suggest other potential fields of elastography application, e.g. plastic breast surgery, radiotherapy or chemotherapy progress, oncologic complication and rehabilitation after breast cancer treatment [1].

Almost all properties of breast tissue are associated with diseased tissue, like water content, acoustic tissue scattering and density and many physiologic fluctuations [12]. Results of our examinations in normal breast tissue using supersonic shear imaging are similar to those described by Tanter et al. - in normal fat and glandular tissue the Young's modulus ranged between $3 \mathrm{kPa}$ and $45 \mathrm{kPa}$. Mean elasticity in glandular tissue in our study was $11.28 \mathrm{kPa}$, and was lower than mean values measured by Tanter et al. They also noticed smaller values of fat tissue than glandular regions, but this difference in our study was smaller than that reported by Tanter et al. Elasticity of fatty tissue in our results was $9.24 \mathrm{kPa}$ and was similar to values measured by these authors (5-10 kPa) [8]. Our results with lower Young's modulus of fat and glandular tissue are similar to other authors who performed MR (magnetic resonance) elastography measurements $[8,13]$. McKnight et al. used in MR elastography $100 \mathrm{~Hz}$ shear wave and their glandular tissue was characterized by mean Young modulus of $7.6 \pm 3.6 \mathrm{kPa}$. For fat tissue it was $3.3 \pm 1.9 \mathrm{kPa}$ and the heterogeneity was modest. They did not calculate exact values, but reported that fibroglandular tissue had higher heterogeneity values, as in our study [13].

The phenomenon of different Young modules in some studies could be partially explained by the $250 \mathrm{~Hz}$ frequency of shear wave (bandwidth ranging from 50 to $450 \mathrm{~Hz}$ ) compared to $50-80 \mathrm{~Hz}$ in MRI [8].

Other application of elastography could be the ultrasonic evaluation of microcalcifications. Cho et al. analyzed breast elastograms from regions corresponding to mammographic microcalcifi-

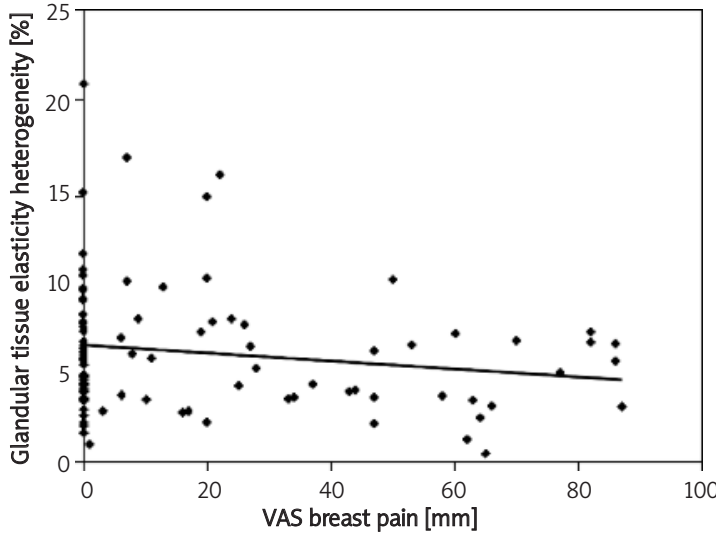

Figure 3. Correlation between glandular tissue elasticity heterogeneity and breast mastalgia measured by VAS

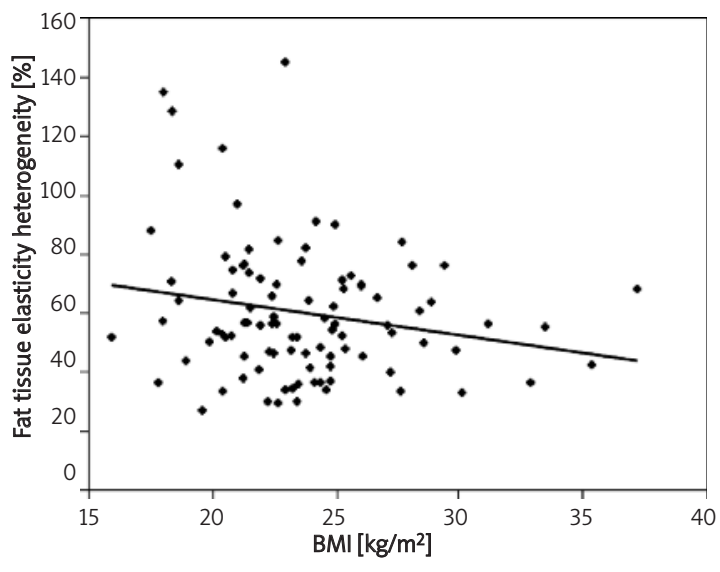

Figure 4. Correlation between fat tissue elasticity heterogeneity and BMI

cations. The breast stiffness was lower in cases of benign conditions when compared with cancers. But the difference was statistically significant when benign nonproliferative, proliferative, in situ cancers were compared with invasive malignant disease. Interestingly, the prediction of benign lesion was $100 \%$ when the microcalcifications region was smaller than $1 \mathrm{~cm}$ in diameter [14].

In some earlier studies, DCIS (Ductal Carcinoma in situ) specimens were measured in vitro and demonstrated more complex elastic modules than normal breast tissue. In empirical studies, DCIS specimens were softer than normal glandular and fibrous tissue at low strain levels and simultaneously stiffer at high strain levels [14, 15]. But these studies were performed using the older technique consisting of breast compression that introduces potentially additional examiner-dependent error. That is why we proposed researching the elasticity measurements by the shear wave technique. Within the Q-box, the circular area of the elasticity map comprises many pixels representing exactly measured elasticity. We called this parameter 
tissue heterogeneity. The fat tissue heterogeneity correlated with BMI and glandular elasticity heterogeneity correlated with mastalgia. Knowledge of exact values of stiffness and physiological fluctuations is of potential use. Mcknight et al. showed that patients with lower tumour stiffness measurements tended to have lower shear stiffness in adjacent adipose tissue. Patients with tumours of higher stiffness had also higher adjacent fat tissue measurements. This could occur due to local oedema [13]. As the physiologic conditions were estimated in our pilot study, further analyses with breast pathologic conditions are needed to evaluate the potential use of elastographic tissue heterogeneity.

The correlation between viscoelastic properties of breast tissue and factors which influence physiologic changes (such as age, menstrual cycle, hormonal status, lactation and others) is a new problem and little is known about it. Many physiological changes depend on the age, hormonal status, menstrual cycle and many others [16]. The use of hormonal contraception, age, pregnancy are also very important for histological changes in the breast, like fibroglandular proliferation and apoptosis [17, 18]. Various hypotheses were developed to explain the observed variation of breast volume and hydratation. The same process occurs during pregnancy and to a lesser degree, during every menstrual period. Especially pregnancy causes an increase in the hyperplasia of epithelial cells, their differentiation and organization into ducts, and the disappearance of connective tissue. The reverse occurs when lactation is interrupted, epithelial cells disappear and connective tissue goes hyperplasic $[16,19]$. The question is why we observed a positive correlation between age and breast elasticity (stiffness) in the whole breast and especially in inner quadrants compared to outer quadrants. Why does the elasticity differ between quadrants? The probable answer could be partially explained by breast composition. There is growing evidence that breast cancers in inner quadrants have increased mortality [20, 21]. Sarp et al. observed lower grading of breast cancers in outer quadrants (G1, 37.7\% vs. $25.4 \%$ ) and this is the reason why inner quadrants cancer have poorer prognosis. As a consequence, the percentage of internal mammary chain metastases increase [20]. Tumours in outer quadrants are also more frequent and it was believed to be directly proportional to the amount of breast tissue in each quadrant. But this hypothesis of tumour development solely on the tissue volume does not explain much higher excess of cancers in outer quadrants. Ellsworth et al. observed greater genomic instability in outer quadrants compared to inner ones. This could partially explain the tendency for tumours to develop in outer quadrants and not simply as a function of breast tissue volume differences [21]. This could support the hypothesis of differences in inner and outer breast quadrants tissue composition, activity and hormonal regulation. But little is known how it influences breast elasticity in inner and outer quadrants. We could only speculate that agedependent correlation between glandular tissue and elasticity depends on faster glandular tissue remodelling in inner quadrants. But it remains unknown why lactation influences fatty tissue heterogeneity. The number of pregnancies and age of the first pregnancy did not influence the elasticity and its heterogeneity, probably because of more subtle cell changes rather than tissue remodelling. But in other studies it has been observed that breast mammographic density decreases with age and pregnancies. It was correlated with water breast content. Empirical evidence and Pike model of breast cancer development emphasize an important role of breast-tissue composition especially in younger subjects [2]. The design of our study included patients at different ages, not only in perimenopause.

Mammography and MRI are different methods of density evaluation, but are well correlated. The mean percentage of breast water that represents fibroglandular tissue differs especially in younger women and this difference decreases with age. However, women with more dense tissue at a young age have a higher risk for cancer [2]. Breast elasticity measured in our study is probably also independent of pregnancy-dependent factors and more correlated with family-specific physical features of breasts. Further studies comparing and correlating elastography with mammography, MRI, risk factors are needed.

Summarizing, the quantitative mapping of breast tissue elasticity is feasible in vivo when using the shear wave technology. This newly developed elastography mode is promising to be less operatordependent than previously used static elastography. In the first case, the remote excitation is produced by the system itself and not by mechanical displacement by the operator [9-12]. All these parameters and their derivates like heterogeneity could add new clinical values in various fields of breast physiology, pathology and treatment.

In conclusion, we estimated that glandular tissue elasticity measured as Young modulus by shear wave ultrasound correlates positively with age. Fatty tissue elasticity correlates positively with duration of lactation. Heterogeneity of the elasticity map of glandular tissue correlates with breast pain and fat tissue with BMI. 


\section{References}

1. Gefen A, Dilmoney B. Mechanics of the normal woman's breast. Technol Health Care 2007; 15: 259-71.

2. Boyd N, Martin L, Chavez S, et al. Breast-tissue composition and other risk factors for breast cancer in young women: a cross-sectional study. Lancet Oncol 2009; 10: 596-80.

3. Soman SD, Joseph KT, Raut SJ, Mulay CD, Parameshwaran $M$, Pandy VK. Studies on major-trace element content in human tissues. Health Phys 1970; 19: 641-56.

4. Lorenzen J, Sinkus R, Biesterfeldt M, Adam C. Menstrualcycle dependence of breast parenchyma elasticity: estimation with magnetic resonance elastography of breast tissue during the menstrual cycle. Invest Radiol 2003; 38: 236-40.

5. Oliver A, Llado X, Perez E, Pont J, Denton ER, Freixenet J, Marti J. A statistical approach for breast density segmentation. J Digit Imaging 2009; 9: 217.

6. Ducote JL, Molloi S. Quantification of breast density with dual energy mammography: a simulation study. Med Phys 2008; 35: 5411-18.

7. Sinkus R, Tanter M, Xydaes T, Catheline S, Bercoff J, Fink M. Viscoelastic shear properties of in vivo breast lesions measured by MR elastography. Magnetic Res Imag 2005; 23: 159-65.

8. Tanter M, Bercoff J, Athanasiou A, et al. Quantitative assessment of breast lesion viscoelasticity: initial clinical results using supersonic shear imaging. Ultrasound Med Biol 2008; 9: 1373-86.

9. Itoh A, Ueno E, Tohno E, et al. Breast disease: clinical application of US elastography for diagnosis. Radiology 2006; 239: 341-50.

10. Thomas A, Fischer T, Frey H, et al. Real-time elastography - an advanced method of ultrasound: first results in 108 patients with breast lesions. Ultrasound Obstet Gynecol 2006; 28: 335-40.

11. Hiltawsky KM, Kruger M, Starke C, Heuser L, Ermert H, Arne J. Freehand ultrasound elastography of breast lesions: clinical results. Ultrasound Med Biol 2001; 27: 1461-9.

12. Konofagou EE. Quo vadis elasticity imaging? Ultrasonics 2004; 42: 331-6.

13. McKnight AL, Kugel JL, Rossman PJ, Manduca A, Hartmann LC, Ehman RL. MR elastography of breast cancer: preliminary results. Am J Roentgen 2002; 178: 1411-7.

14. Cho N, Moon WK, Park JS. Real-time elastography in the differentiation of suspicious microcalcifications on mammography. Eur Radiol 2009; 19: 1621-8.

15. Krouskop TA, Wheeler TM, Kallel F, Garra BS, Hall T. Elastic moduli of breast and prostate tissues under compression. Ultrason Imaging 1998; 20: 260-74.

16. Hussain Z, Roberts N, Whitehouse GH, Garcia-Finana M, Percy D. Estimation of breast volume and its variation during the menstrual cycle using MRI and stereology. Br J Radiol 1999; 72: 236-45.

17. Going JJ, Anderson TJ, Battersby S, Macintyre CCA. Proliferative and secretory activity in human breast during natural and artificial menstrual cycle. Am J Pathol 1988; 130: 193-204.

18. Potten CS, Watson RJ, Wiliams GT, Tickle S, Roberts SA, Harris M, Howell A. The effect of age and menstrual cycle upon proliferative activity of the normal human breast. Br J Cancer 1988; 58: 163-70.

19. Yakut Zl, Kafali H, Karaoglanoglu M, Koktener A, Duvan Cl. A new radiological approach to cyclic mastalgia: venous Doppler ultrasound. The Breast 2009; 18: 123-5.

20. Sarp S, Fioretta G, Verkooijen HM, Vlastos G, Rapiti E, Schubert H, Sappino AP, Bouchardy C. Tumor location of the lower-inner quadrant is associated with an impaired survival for women with early-stage breast cancer. Ann Surg Oncol 2007; 14: 1031-9.

21. Elsworth DL, Elsworth RE, Love B, Deyarmin B, Lubert SM, Mittal V, Hooke JA, Shriver CD. Outer breast quadrants demonstrate increased levels of genomic instability. Ann Surg Oncol 2004; 11: 861-8. 